\title{
Sufficient Autonomy and Satiable Reasons ${ }^{1}$
}

\author{
ROBERT HUSEBY \\ University of Oslo
}

\begin{abstract}
In this paper, I examine Liam Shields' principle of sufficient autonomy. This principle is in many ways interesting and plausible, but it is also in some important respects inadequately specified. In particular, I argue that a) the role of satiable reasons should be clarified, b) the relation to the conditions of freedom should be made more explicit, and c) the threshold for sufficient autonomy should be specified.
\end{abstract}

Keywords: autonomy; conditions of freedom; satiability; shift sufficientarianism; sufficientarianism

\section{INTRODUCTION}

In his recent book Just Enough, Liam Shields presents a novel form of sufficientarianism, which he calls shift-sufficientarianism. Most sufficientarian theories accept what Paula Casal (2007:317) has termed the positive and the negative theses. ${ }^{2}$ According to the positive thesis, there is a level of benefits such that it is especially important, from the point of view of distributive justice, that people reach it. According to the negative thesis, further questions of distributive justice do not arise above this level. The negative thesis is controversial, ${ }^{3}$ and Shields sides with critics who hold that it is implausible (Arneson 2005; Casal 2007; Shields 2016). He therefore discards the negative thesis and claims that sufficientarians can make do with a combination of the positive thesis and the shift thesis:

1 An earlier version of this paper was presented at the Nordic Network in Political Theory in Stockholm in 2017. I am grateful to the participants at that event, as well as to the guest editors, and two anonymous reviewers for this journal, for many helpful comments and suggestions.

2 See for instance Axelsen and Nielsen (2015), Benbaji (2005, 2006), Crisp (2003), Frankfurt (1987), Huseby $(2010,2012)$, Nielsen (2016).

3 The extent to which it is controversial depends on where the threshold is set. The lower the threshold, the more controversial the thesis. 
"Once people have secured enough, there is a discontinuity in the rate of change of the marginal weight of our reasons to benefit them further" (2016: 30). ${ }^{4}$

The main advantage of this proposal is that it saves sufficientarians from having to claim that benefits and distribution above the threshold are completely irrelevant. Rather, benefits to the sufficiently well off matter somewhat, but benefits to those below the threshold matter disproportionately more. This view is in some respects similar to prioritarianism. However, it differs from prioritarianism in that the moral value of benefits does not decrease continuously the better off the recipient is. ${ }^{5}$ Rather, there is a break, or a shift, at the sufficiency threshold. ${ }^{6}$

From the formulation of the shift thesis (2016:30), one gets the impression that when distributing some good $\mathrm{G}$ it is especially important that people get a sufficient amount of $\mathrm{G}$ and that, beyond this, it might still be valuable, though less so, to provide people with further G. On this understanding, the reasons we have for supplying $G$ do not change, but the weight of these reasons diminishes. However, it appears that Shields also thinks that the reasons can change altogether at the threshold (2016: 30). On this understanding, it is still important that people receive sufficient G, but after that, further provisions of $\mathrm{G}$, if valuable, are valuable in light of some reason other than the value of G. For instance, G, above the threshold, might lead to the realization of some other good, W. I assume, however, that there will still be a discontinuity in the rate of change of the marginal weight in the cases where the reasons as such change. Otherwise, the shift thesis would not apply to this latter version.?

My aim in this paper is to examine the most specific proposal for a shift sufficientarian principle made by Shields, namely the principle of sufficient autonomy. In my view, this principle is in important respects not sufficiently specified. However, since I am in general sympathetic to the suggestion of a principle of sufficient autonomy, my criticisms are largely calls for clarification and further development of the view.

4 The shift thesis was first presented in Shields (2012: 108).

5 See for instance Holtug (2010: 133).

6 Gustaf Arrhenius has suggested that prioritarianism really is characterized by many small shifts, and that Shields' theory, with only one shift, is a minimal form of prioritarianism. This seems plausible to me, but I will nevertheless treat it as a version of sufficientarianism here.

$7 \quad$ Shields also holds that the shift could come about as a result of conflict between reasons (2016: 39). I leave this alternative aside here 


\section{SATIABILITY AND THE PRINCIPLE OF SUFFICIENT AUTONOMY}

Shields cashes out the shift in terms of satiable reasons, based on Raz' concept of satiable principles.

"Satiable principles are marked by one feature: the demands the principles impose can be perfectly met. When they are completely met then whatever may happen and whatever might have happened the principles cannot be, nor could they have been, satisfied to a higher degree" (Raz 1986: 235f, cited in Shields 2016: 36).

Shields further writes:

"Our reasons to benefit people change when they are no longer deficient in the relevant respect. There may be strong claims for benefits beyond the application of that reason, weneed notbeupperlimitsufficientarians, but such claims must be made using a different profile of reasons. This alters our all things considered reasons to benefit people further" (2016:37).

Shields oscillates, as noted, between suggesting that (only) the weight of the reasons change, and that the reasons themselves (also) change. Nonetheless, in the elaboration of the principle of sufficient autonomy, Shields for the most part writes as if the reasons change altogether. According to this principle, "...we have weighty, non-instrumental, non-egalitarian reasons to secure sufficient autonomy to secure the social conditions of freedom" (2016: 53) ${ }^{8}$ Even though satiability is not incorporated explicitly into this formulation, it is clear that the principle is intended to be satiable (see 2016: $45,50,57$ ).

\section{IS THE PRINCIPLE OF SUFFICIENT AUTONOMY SATIABLE?}

Despite Shields' intentions, it is unclear whether the principle of sufficient autonomy really is satiable. Note that there are two ways of understanding satiability in this context. First, we could think of satiability as applied directly to autonomy (or to whatever value a principle is intended to promote). On this view, autonomy is a satiable concept if one can be fully autonomous. This understanding is indicated by Shields when he writes that "[o]ur reasons to benefit people change when they are no longer deficient in the relevant respect (2016: 37; see also 2012: 118)". He further illustrates sufficiency with reference to "enough sleep", and "enough

8 I return to the social conditions of freedom below 
petrol" (2106: 29), and satiability with reference to "enough money for a bus ticket" (2016: 36). These examples suggest that satiability refers to the value that we should have a sufficient amount of.

Alternatively, satiability might refer to the principle of sufficiency itself (2016: 64). Sufficiency is straightforwardly a satiable principle, since enough is enough. Utilitarianism is not satiable, because there can always be more utility. On this view, sufficient autonomy does not (necessarily) demand full autonomy, but autonomy to some degree that is deemed sufficient for some other reason. Since it is not perfectly clear to me which of these views Shields holds, I will discuss both. I discuss the former in this section, and the latter (more indirectly) in the two subsequent sections.

According to Shields, "[a]utonomy is the ideal of living one's life in accordance with one's own authentic judgments" (2016: 47). Autonomy, moreover, is characterized both by the absence of external pressure or constraints, such as threats, coercion or brainwashing, as well as the presence of options and powers of deliberation. Both aspects are needed for people to be autonomous (2016: 47f). However, Shields also writes:

"To flesh this out we can say that sufficient autonomy has three conditions. One has secured sufficient autonomy when (1) one is wellinformed, (2) one can give reasons for one's views, and (3) one has a disposition to exchange reasons and participate in a public deliberative process with others" (2016: 53).

I focus nevertheless on freedom from coercion, options, and deliberative powers in the following, as these seem more central to the concept of autonomy. After all, one can meet the three fleshed-out conditions above without having any options, and without being free from external constraints. I take it, moreover, that being well-informed, and having the ability to give reasons for one's views can plausibly be subsumed under deliberative powers.

Consider now the different aspects of autonomy in terms of (the first understanding of) satiability. The first, freedom from coercion, does admittedly seem satiable. One can presumably be perfectly free from external pressures and constraints. The second aspect is less clearly satiable. One can always have more options to choose from and one can always gain better powers of deliberation. Thus, there is a sense in which one can never be fully autonomous, and one's claim for autonomy cannot be perfectly sated.

An obvious rejoinder is that there will still be some level at which even more (trivial) options, and even more finely developed powers of deliberation, makes no difference for any practical purposes. Perhaps one 
already has all the options one could possibly want, and perhaps one is able to rank all these options (and their combinations) perfectly on an ordinal scale. Adding options one does not want, or gaining the ability to rank the options and their combinations cardinally, makes no difference, let us assume, to how one leads one's life. In such cases, I agree that autonomy is sated, for practical purposes.

However, if autonomy is satiable, even if only in this practical sense, another question arises. Now it is not clear that further increasing autonomy above the sufficiency level will contribute to people's welfare, or anything else for that matter. If satiable in this way, satiability occurs at such a high level that there does not seem to be any further reason, egalitarian or otherwise, to provide people with even more autonomy. The principle of sufficient autonomy now looks like an upper limit sufficiency principle that conforms to the negative thesis.

One could imagine, though, that a person who is, for practical purposes, perfectly autonomous, can still benefit from more of the stuff that constitutes autonomy, for reasons not to do with autonomy. For instance, a person might get a thrill from gaining access to even more trivial options, even though none of these additional options will be chosen. Or, the person might enjoy even better deliberative powers, for the feeling of being superclever. ${ }^{9}$ However, if so, I am inclined to think that what is provided is not more autonomy as such, but more options or more deliberative powers. To illustrate, suppose you are perfectly nourished. There are, for all practical purposes, no way for you to be even more nourished. Suppose however, that more vitamin D (unrealistically) might provide you with a nice tan. If so, providing you with more vitamin $\mathrm{D}$, does not in that case provide you with more nourishment, it gives you more vitamin D (and a tan). It seems then, that if the principle of sufficient autonomy is satiable (with respect to autonomy), it is likely to be so at such a high level that it is hard to detect a relevant shift.

Consider now the alternative understanding of satiability. Perhaps there is a level of autonomy that is sufficient, regardless of whether or not it is equivalent to full or perfect autonomy. The reasons for promoting someone's autonomy, then, are sated when they reach this level. This might be perfectly reasonable, but the level would have to be specified. As will become clear below, it is hard to find such a specification in Shields' treatment of sufficient autonomy. Let me note, at any rate, that the first kind of satiability has an obvious advantage: It is very useful for defining a threshold. Sufficientarianism is sometimes criticized for the alleged

9 I am grateful to David Axelsen for raising this point. 
arbitrariness of the threshold(s). The first notion of satiability might help meet this objection.

\section{THE SOCIAL CONDIITONS OF FREEDOM}

As noted, Shields ties the ideal of autonomy to the social conditions for freedom in society. However, the link between these two concepts is not entirely clear:

"The principle of sufficient autonomy, supported by the sufficientarian reason we have to live under the social conditions of freedom, can be stated thus: we have weighty, non-instrumental, non-egalitarian reasons to secure sufficient autonomy to secure the social conditions of freedom. The conditions of freedom are those conditions under which one's beliefs and actions can be considered freely taken" (2016: 54).

The last sentence of the quote indicates that the social conditions of freedom are those conditions under which autonomy is possible (or likely, or certain). Both the conditions of freedom and the principle of autonomy, moreover, appear to be sufficientarian concepts, but the former supports the latter. Shields suggests, moreover, that the social conditions for freedom ought to be such that they are sufficient, as opposed to merely necessary, for the development of sufficient autonomy (see also 2016: 45, 48, 53, 54).

However, the first sentence seems to indicate the opposite; that autonomy is a condition for the social conditions of freedom. It could be the case that autonomy and the social conditions of freedom are interdependent in a way that vindicates both views. However, it seems to me more plausible to say that autonomy presupposes certain societal conditions for its realization, than to say that the social conditions for freedom presupposes autonomy for its realization. Shields points to a proper education, an ethos marked by toleration, and reliable information as parts of the conditions of freedom (2016: 53). These factors are more plausibly seen as conditions for the development of autonomy, than the other way around.

However, even if we accept this, there is a further option. There are formulations that suggest that autonomy is itself (the whole or a part of) the social conditions of freedom: "...I set out and provide an initial defence of the account of autonomy that constitutes the conditions of freedom" (2016: 46). Further: "One reason for promoting individual autonomy is our interest in the conditions of freedom. It is a weighty, non-egalitarian reason that is satiable with respect to autonomy, at least" (2016: 57 ). The last quote gives the impression that autonomy is one (satiable) part of what constitutes the 
conditions of freedom (see also 2016: $57,58,60$ )

I confess to being unable to determine whether the social conditions of freedom are to be understood as those conditions that are (necessary and) sufficient for the realization of sufficient autonomy, or whether sufficient autonomy constitutes (or is a condition for) the social conditions for freedom. However, the question is important for how we interpret the principle of sufficient autonomy as a satiable sufficientarian principle.

The reason is that if autonomy is a part of what constitutes the conditions of freedom (or if it is a condition of freedom in itself), then it could be the case that autonomy can be sated with respect to the conditions of freedom. Sufficient autonomy just is autonomy sufficient for the realization of (sufficient) conditions of freedom. However, in my view, this interpretation squares badly with Shields' presentation of the principle, according to which there are supposed to be weighty, non-instrumental, satiable reasons to provide peoples with sufficient autonomy (2016: 45). There might be ways to interpret autonomy as non-instrumentally valuable even if it is a constitutive part of the conditions of freedom. But this is not stated explicitly, and would require further elaboration and specification.

\section{THE SUFFICIENY THRESHOLD}

In this section, I consider the way Shields specifies the threshold for sufficient autonomy (regardless of how satiability is understood, and on the assumption that the threshold is not determined in light of the demands of the conditions of freedom). Note first that there are several formulations concerning this threshold in the book, and that not all of them point in the same direction. However, what is perfectly clear is that Shields assumes that there is a level at which we can say that a person has sufficient autonomy, and that there might, because of the shift that occurs at this level, be further reasons to promote the autonomy of those who already have sufficient autonomy (2016: 54).

Shields offers an example of the importance of sufficient autonomy with reference to the information needed to assess the risks associated with buying a house.

"If we do not know the risk, but we know how to find out about it, we might think that we choose freely ... If we are well informed enough to become well informed about the other relevant decisions we make, then our reasons to become more and more well informed thereafter may be very different" (2106: 53). 
The latter part of the quote, obviously, indicates a shift. However, it is not clear how this works. Suppose I face a risk. I do not know the risk exactly, but I do know how to calculate it (or as Shields suggests, I have the contact details of a surveyor that can help me assess the risk). In this situation, I am not as autonomous as I can be. But I am free to become as autonomous as I can be (with regards to this particular decision). It is up to me whether to pick up the phone or start punching numbers into the calculator. Something similar can be said about the "well-informed enough to become well-informed" part of the quote.

The shift, on the view suggested here, is located at the level at which we are sufficiently autonomous to choose to become fully autonomous. This might be plausible, but it seems that this amounts to a view of sufficiency that is, in a relevant sense, an upper limit view, of the sort Shields aims to avoid. One might say, of course, in line with the shift thesis, that the reasons to promote a person's autonomy above the level at which it is up to the person herself to become fully autonomous, change. But more likely, they disappear. For all practical purposes, one is fully autonomous when one has direct access to becoming fully autonomous. It makes no sense to benefit a person, in terms of autonomy, because she has not decided to punch the numbers, place the call, or perhaps consult the relevant literature. There is no longer a recognizable distributive issue to be addressed.

Another view is suggested by the following: "Only once an agent is autonomous can we fully respect his or her answer to the question 'Do you want to enhance your autonomy?' We owe them autonomy sufficient for making these kinds of choices freely as part of justice" (2016: 56). While it is certainly true that people should be (at least) sufficiently autonomous to know whether they want to become more autonomous, it is unlikely that people are sufficiently autonomous from the point of view of justice, at this exact level. The reason is that this level might be quite low, at least along some relevant dimensions. For instance, people who are severely oppressed, and have very few options, may well be more than sufficiently autonomous for us to respect their wish to become more autonomous. The alternative is clearly disrespectful. Notice that the level of autonomy that Shields points to here might (or might not) mark a relevant shift, but not a shift that signifies sufficient autonomy. ${ }^{10}$

Further, Shields claims that

"Sufficient autonomy is the level of deliberative competence that enables us to have assurance from an external point of view that we

10 For a similar suggestion, though in more convoluted terms, see Shields (2016: 57). 
choose for ourselves. This kind of autonomy requires us to be capable of deliberating with others about the reasons that support our conception of the good. We may have weighty reasons to secure more autonomy..." (2016: 64).

The first part of the quote points to one intuitively important and plausible aspect of autonomy, namely self-rule. However, it is unclear what level exactly it refers to. What does it take to be sure that a person chooses for herself? And what aspects of autonomy is it that can be further promoted, but for different reasons? It is unclear what level of freedom from constraints and access to options, for instance, are also required, since these aspects are not mentioned. The notion of choosing for oneself, then, does not point to a clearly discernible level of sufficient autonomy.

In other passages, Shields suggests that ambition, and our conceptions of the good may influence the level of autonomy that we want, but that the level of sufficient autonomy is independent of such ambitions and conceptions (2016: 65). A similar view is suggested in the case of Agnes and Bernadette (see 2016: 70f). Bernadette supposedly has sufficient autonomy, because the reasons we might have to promote her autonomy further are instrumental with regards her welfare, and not non-instrumental with regards to her autonomy. On Shields' description, she has "many options", the "ability to make medium- and long-term plans, can usually spot contradictions in her own judgments and can remedy them", "makes reasoned assessments of various ways of life, and is not being denied information about the costs and benefits of her choices" (2016: 70).

This arguably offers some substance, but it is still not clear enough. I will not detail all the ways in which these different elements are less than perfectly specific. However, I think it is worth pointing out that the subsequent claim that our reasons to boost Bernadette's autonomy further is dependent on whether or not it can contribute to her well-being, suggests a fairly high level of autonomy (2016: 71). The reason is that the level at which more autonomy does not have non-instrumental value sufficient for speaking in favor of further promotion by itself, appears to be high. It would be good, of course, to know how high.

\section{CONCLUDING REMARKS}

The principle of sufficient autonomy might turn out to be a valuable and plausible principle. However, at present, I think that the principle needs further specification when it comes to the understanding of satiability, the relation to the conditions of freedom, and the location of the threshold. In 
closing, I would like to add a fourth call for clarification: the principle of sufficient autonomy is clearly only one among several principles of justice. Shields suggests that there might be many others, and that not all of them need to be sufficientarian. However, in the book, it is hard to get a clear view of the larger picture. It would be interesting to learn more about how these different principles relate to each other and form a coherent general theory of distributive justice.

\section{BIBLIOGRAPHY}

Arneson, R., 2005: “Distributive Justice and Basic Capability Equality: 'Good Enough' is not Good Enough," in Capabilities Equality: Basic Issues and Problems, ed. A. Kaufman, 17-43, London: Routledge.

Axelsen, D. and L. Nielsen., 2015: “Sufficiency as Freedom from Duress,” The Journal of Political Philosophy 23: 406-426.

Benbaji, Y., 2005: “The Doctrine of Sufficiency: A Defence,” Utilitas 17: 310-332.

Benbaji, Y., 2006: “Sufficiency or priority?” European Journal of Philosophy 14: 327-348.

Casal, P., 2007: "Why Sufficiency Is Not Enough,” Ethics 117: 296-336.

Crisp, R., 2003: “Equality, Priority, and Compassion,” Ethics 113: 745-763.

Frankfurt, H., 1987: “Equality as a Moral Ideal," Ethics 98: 21-43.

Holtug, N., 2010: Persons, Interests, and Justice, Oxford: Oxford University Press.

Huseby, R, 2010: "Sufficiency - Restated and Defended." The Journal of Political Philosophy 18: 178-197.

Huseby, R., 2012: “Sufficiency and Population Ethics," Ethical Perspectives 19: 187-206.

Nielsen, L., 2016: "Sufficiency Grounded as Sufficiently Free: A Reply to Shlomi Segall," Journal of Applied Philosophy 33: 202-216.

Raz, J., 1986: The Morality of Freedom, Oxford: Oxford University Press.

Shields, L., 2012: “The Prospects for Sufficientarianism,” Utilitas 24: 101-117.

- 2016: Just Enough: Sufficiency as a Demand of Justice, Edinburgh: Edinburgh University Press. 